\title{
Article \\ The Genomic Landscape of a Restricted ALL Cohort from Patients Residing on the U.S./Mexico Border
}

\author{
Alice Hernandez Grant ${ }^{1}{ }^{\mathbb{D}}$, Yoshira Marie Ayala-Marin ${ }^{1}$, Jonathon Edward Mohl ${ }^{2} \mathbb{D}$, Elisa Robles-Escajeda ${ }^{1} \mathbb{D}$, \\ Georgialina Rodriguez ${ }^{1}$, Julie Dutil ${ }^{3}$ and Robert Arthur Kirken ${ }^{1, *}$ \\ 1 Department of Biological Sciences, College of Science, The University of Texas at El Paso, \\ El Paso, TX 79968, USA; aehernandez6@utep.edu (A.H.G.); ymayala2@utep.edu (Y.M.A.-M.); \\ erobles3@utep.edu (E.R.-E.); grodriguez@utep.edu (G.R.) \\ 2 Department of Mathematical Sciences, College of Science, The University of Texas at El Paso, \\ El Paso, TX 79968, USA; jemohl@utep.edu \\ 3 Department of Biochemistry, Cancer Biology Division, Ponce Research Institute, \\ Ponce Health Sciences University, Ponce, PR 00716, USA; jdutil@psm.edu \\ * Correspondence: rkirken@utep.edu; Tel.: +1-(915)-747-5536
}

check for

updates

Citation: Grant, A.H.; Ayala-Marin, Y.M.; Mohl, J.E.; Robles-Escajeda, E.; Rodriguez, G.; Dutil, J.; Kirken, R.A. The Genomic Landscape of a Restricted ALL Cohort from Patients Residing on the U.S./Mexico Border. Int. J. Environ. Res. Public Health 2021, 18, 7345. https://doi.org/ 10.3390/ijerph18147345

Academic Editors: Paul B. Tchounwou, Daniel F. Sarpong, Elizabeth O. Ofili, Emma

Fernandez-Repollet, Richard J. Noel, Magda Shaheen, Kristen J. Wells and Richard Yanagihara

Received: 1 April 2021

Accepted: 6 July 2021

Published: 9 July 2021

Publisher's Note: MDPI stays neutral with regard to jurisdictional claims in published maps and institutional affiliations.

Copyright: (c) 2021 by the authors. Licensee MDPI, Basel, Switzerland. This article is an open access article distributed under the terms and conditions of the Creative Commons Attribution (CC BY) license (https:// creativecommons.org/licenses/by/ $4.0 /)$.

\begin{abstract}
Next-generation sequencing (NGS) has identified unique biomarkers yielding new strategies in precision medicine for the treatment of Acute lymphoblastic leukemia (ALL). Hispanics show marked health disparities in ALL, often absent in clinical trials or cancer research. Thus, it is unknown whether Hispanics would benefit equally from curated data currently guiding precision oncology. Using whole-exome sequencing, nine ALL patients were screened for mutations within genes known to possess diagnostic, prognostic and therapeutic value. Genes mutated in Hispanic ALL patients from the borderland were mined for potentially pathogenic variants within clinically relevant genes. KRAS G12A was detected in this unique cohort and its frequency in Hispanics from the TARGET-ALL Phase II database was three-fold greater than that of non-Hispanics. STAT5B $\mathrm{N} 642 \mathrm{H}$ was also detected with low frequency in Hispanic and non-Hispanic individuals within TARGET. Its detection within this small cohort may reflect a common event in this demographic. Such variants occurring in the MAPK and JAK/STAT pathways may be contributing to Hispanic health disparities in ALL. Notable variants in ROS1, WT1, and NOTCH2 were observed in the ALL borderland cohort, with NOTCH2 C19W occurring most frequently. Further investigations on the pathogenicity of these variants are needed to assess their relevance in ALL.
\end{abstract}

Keywords: ALL; relapse; precision medicine; Hispanic health disparities; KRAS; STAT5B; NOTCH2; ROS1; WT1

\section{Introduction}

Acute lymphoblastic leukemia (ALL) is a malignant transformation of lymphoid precursor cells commonly occurring in children. Frontline therapies, typically multi-agent chemotherapy strategies, have led to remission in $95 \%$ of pediatric ALL. However, a subgroup does exhibit refractory or ALL relapse, and the five-year free survival rate is reduced to $15-50 \%$ [1]. This subgroup is disproportionately reported for Hispanic children who experience a 5\% lower five-year free survival rate for ALL than their non-Hispanic counterparts [2]. The etiology for ALL relapse is unclear, but advances in stratification based on chromosomal abnormalities and genetic alterations are guiding their prognosis and treatment management [3]. These improvements in stratification are largely derived from next-generation sequencing (NGS) studies that have revealed unique biomarkers associated with diagnostic criteria as well as prognostic relevance of leukemias, yielding to the revised 2016 classifications of acute leukemias by the World Health Organization [4]. These classifications involve chromosomal translocations/fusions that are common in ALL; while others such as Ph-like ALL that are negative for example the BCR-ABL1 fusion rely 
on the detection of kinase-activating aberrations, including rearrangements, mutations, and copy number alterations [5]. In other ALL subtypes, these are observed as co-operating secondary aberrations [6]. In acute myeloid leukemia (AML), genetic aberrations have practical value in treating AML [7] and similarly, ALL genetic aberrations detected by NGS [8] have improved ALL treatment. NGS will continue to influence the diagnosis and prognosis of acute leukemias as clinical oncology progresses toward precision medicine. These efforts are especially needed for improving treatment regimens for refractory and relapse leukemias, including ALL.

It is unknown whether Hispanic patients benefit equally from precision oncology due to a lack of inclusion or participation of minorities in cancer research $[9,10]$. For example, in Texas, estimates of less than $2 \%$ of genome-wide studies on biospecimens are obtained from Hispanics [11]. Therefore, well-known cancer mutations that are principally derived from large databases may or may not reflect what is observed in under-represented MexicanAmerican Hispanic cancer patients. Furthermore, it is unknown whether clinically relevant genes are responsible for driving ALL in this population. To explore the mutational landscape involved in ALL, we performed NGS on ALL patients from the Paso del Norte region with a demographic of $83 \%$ Hispanic, $96 \%$ of Mexican origin. This region is associated with a higher incidence of ALL and inferior survival in Hispanics [12]. Using whole-exome sequencing (WES), the genetic profile of nine ALL patients (four new-onset and five relapse cases) was investigated for mutations within genes having diagnostic, prognostic, and therapeutic value [13]. Next, genes mutated in ALL patients were mined for variants with pathogenic potential [14]. The resulting genes were then screened for their presence across ALL targetable pathways and their occurrence in the Therapeutically Applicable Research to Generate Effective Treatments (TARGET)-ALL Phase II database [15]. The recognized pathogenic variants, KRAS G12A and STAT5B N642H were detected in the ALL borderland cohort. Perhaps, alterations in the mitogen-activated protein kinase (MAPK) and the Janus kinase/signal transducer and activator of transcription (JAK/STAT) pathways are contributing to Hispanic health disparities in ALL. The NOTCH2 C19W variation was a frequent event in the ALL borderland cohort, yet it was not present in the TARGET-ALL Phase II database. The occurrence of this variant in a biomarker gene, along with others mentioned herein, warrant investigation of their contribution to oncogenesis, given their potential relevance in clinical applications. Further NGS investigations in diverse and geographically distinct groups burdened by high incidence, prevalence, and mortality may yield novel variants driving ALL. These efforts have the potential to contribute to guidelines for the stratification of disease and improved treatment.

\section{Materials and Methods}

\subsection{Participants and Sequencing}

All research utilizing human subjects was approved by The University of Texas at El Paso Institutional Review Board (UTEP IRB) committee, and all participants provided written informed consent. Genomic DNA was collected using Puregene Kit A (Qiagen, Germantown, MD, USA) according to the manufacturer's instructions from a small cohort of 7 healthy controls and 9 patients diagnosed with ALL in the El Paso, Texas region. Purified DNA was sent to the Otogenetics Corp. in Atlanta, GA, USA, for WES.

\subsection{Bioinformatics}

Sequences were quality trimmed, mapped to the Gchr38.p1 human reference genome, and genotyped using process_sequences.py (https://github.com/jonmohl/PopGen) (accessed on 1 April 2021) [16-19]. Analysis of the aligned WES data was performed by the Border Biomedical Research Core (BBRC) Bioinformatics unit using OncoMiner [20] to identify non-synonymous amino acid (AA) mutations [21]. Categorization of variants into germline and somatic was not possible due to the absence of matched healthy tissues. To overcome this limitation, single-nucleotide polymorphisms (SNPs) found within coding regions and not present within control samples were extracted and further analyzed. The remaining 
SNPs were then filtered using the GnomAD database in which any position that had a presence of more than 5\% in the Latino/Admixed American group was additionally removed. This somewhat liberal approach was meant to include mostly somatic mutations while enabling the detection of rare germline mutations that may be relevant to cancer in this population. The total number of mutations per sample was compiled. Filtering and summary steps were performed using in-house Python3 scripts. Data compiled from Otogenetics (Gchr37), filtered for SNPs from select genes (96), can be downloaded from the UTEP Bioinformatics Repository (https:/ / datarepo.bioinformatics.utep.edu/getdata?acc=B4U5YED0W0RIE3C) (accessed on 7 July 2021). The ethnicity of the ALL patient samples was determined by analysis of the germline variants obtained from WES data. The WES vcf files were intersected with dbSNP common variants (dbSNP138) [22] and mapped in reference to 1000 Genomes [23] populations using the first 2 dimensions of multidimensional scaling analysis [24]. The control participants self-identified as Hispanic (5) and non-Hispanic (2).

\subsection{Gene Inclusion and Classification of Mutations}

Gene lists with diagnostic, prognostic, and therapeutic value were obtained from OncoKB [13]. OncoKB categorizes genes by diagnostic, prognostic, and therapeutic levels of evidence [13]. Levels 1-4 are defined as follows: an FDA-recognized biomarker (Level 1), standard care biomarker (Level 2), compelling clinical evidence biomarker (Level 3), and compelling biological evidence biomarker (Level 4), where all are found with slight variation across each category. For simplicity, Levels 1-4 are referred to across diagnostic and prognostic evidence, although in OncoKB, this terminology is reserved for therapeutic indications. Therapeutic biomarkers have additional levels that are predictive of drug resistance. From this list of potentially actionable genes, those observed in new-onset and relapse ALL patients were further screened for pathogenic mutations using the Cancer Mutation Census (CMC) within the Catalogue of Somatic Mutations in Cancer (COSMIC) database [14]. Mutations found in the ALL cohort were classified according to their mutational significance in cancer ranked by tier. The $\mathrm{CMC}$ ranks the AA variations in three tiers, depending on the evidence supporting their role in cancer pathogenesis. Three factors that affect their rank include (1) recurrent mutations in Census (CGC) oncogenes or tumor suppressors (nonsense, large indel, and frameshift), (2) pathogenic status in Clinvar, and (3) evidence of positive selection determined by the $\mathrm{dN} / \mathrm{dS}$ ratio. Tier 1 mutations meet factors 1 and 2. Tier 2 mutations have a combination of any two factors 1,2 or 3 . Tier 3 meets at least one of the factors. All other mutations in CGC are ranked as other. Here, we included an additional rank referred to as Tier X, including variants that occur in AA locations that correspond with Tier 1, Tier 2, and Tier 3; yet, the change in AA is not reported in the COSMIC database or labeled as other. Finally, genes containing Tier 1, Tier 2, Tier 3, or Tier X mutations were searched for in targetable pathways, as referenced in My Cancer Genome [15]. All relevant variants were searched against TARGET-ALL Phase II (phs000218) vcf files from white non-Hispanic (408) and white Hispanic individuals (145) to assess their frequency in a larger cohort.

\section{Results}

\subsection{Patient Demographics}

To explore the mutational landscape of Hispanic ALL patients, WES was performed on nine ALL patients from the borderland of the Paso Del Norte region. Patient demographics are shown for new-onset or relapsed ALL patients (Table 1) diagnosed with either T or B cell lineage subtypes. 
Table 1. Patient information.

\begin{tabular}{ccccc}
\hline Patient & Diagnosis & Subtype & Status & Ethnicity \\
\hline P1 & ALL & T-ALL & Relapse & Hispanic \\
P2 & ALL & pre-T-ALL & Relapse & Hispanic \\
P3 & ALL & pre-T-ALL & New onset & Hispanic \\
P4 & ALL & pre-T-ALL & New onset & Hispanic \\
P5 & ALL & early pre-B-ALL & Relapse & Hispanic \\
P6 & ALL & early pre-B-ALL & Relapse & Hispanic \\
P7 & ALL & pre-T-ALL & Relapse & Hispanic \\
P8 & ALL & early pre-B-ALL & New onset & Hispanic \\
P9 & ALL & early pre-B-ALL & New onset & Hispanic \\
\hline
\end{tabular}

\subsection{The Genomic Landscape of ALL}

Using WES, the gene mutation profile of the nine ALL patients was investigated for mutations within clinically relevant genes, including those with diagnostic, prognostic, and therapeutic value. This list of clinically relevant genes (Supplementary Figure S1) was further screened for potential variants with mutational significance. Each variant was ranked by a tier indicative of mutational significance, and, hence, potential cancer pathogenicity. Tier 1 has the highest level of mutational significance and is considered pathogenic in cancer. Tier 2 and Tier 3 have some level of mutational significance and are likely pathogenic in cancer. Tier $\mathrm{X}$ is suspected of having mutational significance; its contribution to cancer is unknown. Each variant was subsequently categorized by its level of clinical relevance corresponding to the particular gene.

\subsubsection{ALL Borderland Patients Harbor Tier Mutations Detected in First-Level Cancer Biomarkers with Therapeutic Potential}

Detected from the ALL borderland cohort were ROS1, ATM, and KRAS; genes with the highest level of clinical significance that were found harboring variants with mutational significance in Tier 3, Tier X, and Tier 1, respectively (Figure 1). These genes are FDA-recognized biomarkers with therapeutic indications in various cancers. Although such indications are not yet applied to ALL, mutations in these genes can contribute to hematological malignancies. For example, ROS1 is a receptor tyrosine kinase (RTK) that can translocate to form oncogenic fusions or express activating aberrations in some hematologic malignancies [25,26]. It is unknown if any ALL patients from our cohort contained fusions in ROS1. The DNA damage response gene ATM is inactivated in various hematological malignancies [27]. A similar variant (ATM E2366 ${ }^{*}$ ) can propagate mutations associated with predispositions to cancer [28]. Lastly, mutations in the GTPase KRAS are prevalent in ALL and are associated with resistance and inferior survival [29]. The mutation frequency of the KRAS hotspot at position 12 is commonly seen in ALL relapse [30].
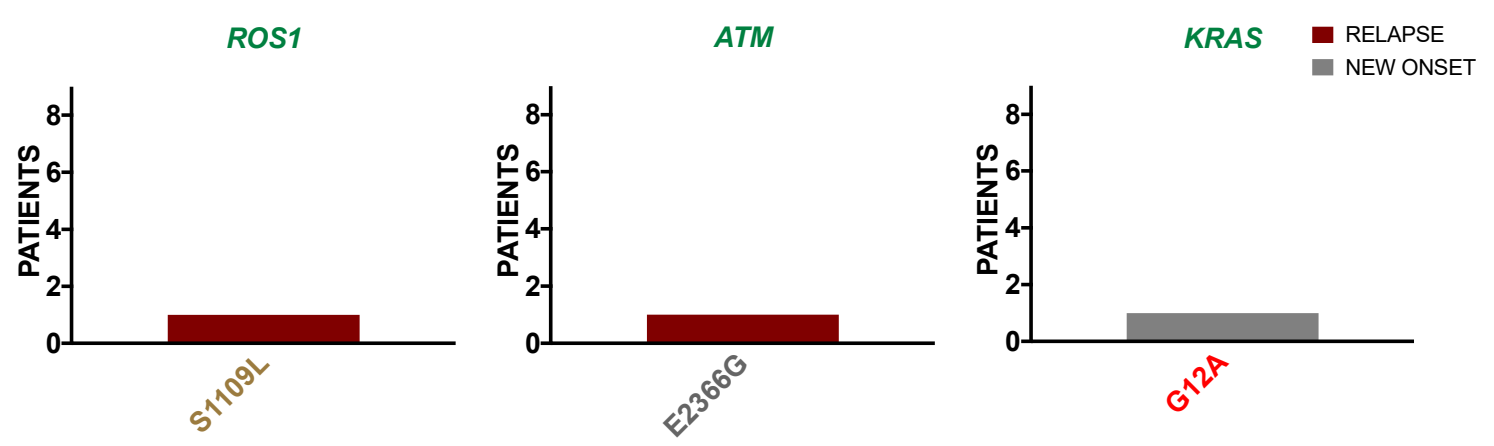

Figure 1. ALL borderland patients harbor tier mutations detected in first level cancer biomarkers with therapeutic potential. Variants are shown occurring in new-onset (grey) and relapse (maroon) ALL patients where mutational significance is indicated by Tier 1 (red), Tier 3 (gold), and Tier X (grey) within Level 1 clinically relevant genes (green) including ROS1, ATM, and KRAS. 
3.2.2. ALL Borderland Patients Harbor Tier Mutations Detected in Second-Level Biomarkers with Diagnostic and Prognostic Value for Hematologic Malignancies

Detected in the ALL borderland cohort were genes involved in epigenetic regulation at the next level of clinical significance, including DNMT3A, TET2, and WT1. These genes were observed harboring variants with mutational significance in Tier X and Tier 3 , respectively (Figure 2). At this second level, DNMT3A, TET2, and WT1 are FDA-recognized biomarkers supporting diagnostic and prognostic indications in hematologic malignancies, excluding ALL. However, lower-level indications for these biomarkers are applicable to ALL prognoses. For example, DNMT3A mutations are associated with relapse and inferior survival outcomes in ALL [31]. Similarly, TET2 and WT1 aberrations are associated with poor prognosis in AML [32,33] and perhaps ALL [34,35]. The associated outcomes are partly due to the loss or interference of epigenetic regulation [36].
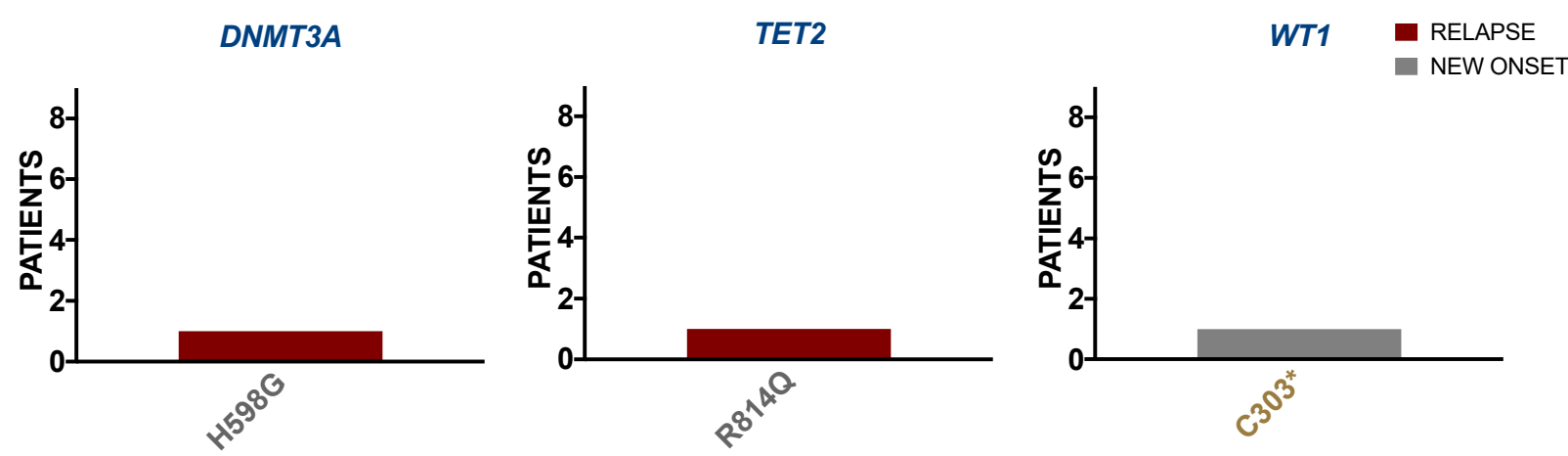

Figure 2. ALL borderland patients harbor tier mutations detected in second-level biomarkers with diagnostic and prognostic value for hematologic malignancies. Variants are shown occurring in new-onset (grey) and relapse (maroon) ALL patients where mutational significance is indicated by Tier X (grey) and Tier 3 (gold) within Level 2 clinically relevant genes (blue) including DNMT3A, TET2, and WT1 involved in epigenetic regulation.

3.2.3. ALL Borderland Patients Harbor Tier Mutations Detected in Third-Level Biomarkers with Diagnostic Value for Leukemia

Detected from the ALL borderland cohort were STAT5B, SUZ12, PTEN, NOTCH2, and SOCS1; genes at the lowest level of clinical significance, that were found harboring variants with mutational significance (Figure 3). Variants in STAT5B and SOCS1 represent alterations in the JAK/STAT pathway (Figure 3a). The SUZ12 variant represents another alteration in epigenetic genes (Figure 3b), as demonstrated in Figure 2. The PTEN variations represent alterations in the PI3K signaling pathway (Figure 3c). Lastly, the enrichment and frequency of NOTCH2 variants in this ALL cohort indicated aberrations in NOTCH2 signaling (Figure 3d). STAT5B and SUZ12 at this level hold diagnostic value for T cell leukemias, while the remaining are relevant to other hematologic malignancies. There is evidence to support a role for these genes in ALL. For example, the activating STAT5B $\mathrm{N} 642 \mathrm{H}$ mutation found in T cell ALL has been associated with risk of relapse [37]. SUZ12 has been considered a tumor suppressor gene mutated in T-ALL [38]. Similarly, PTEN and NOTCH2 mutations have been reported in T-ALL cases [39,40]. Lastly, changes in SOCS1 expression has been reported in refractory AML [41]. 
(a) STAT5B

(b)

sUZ12

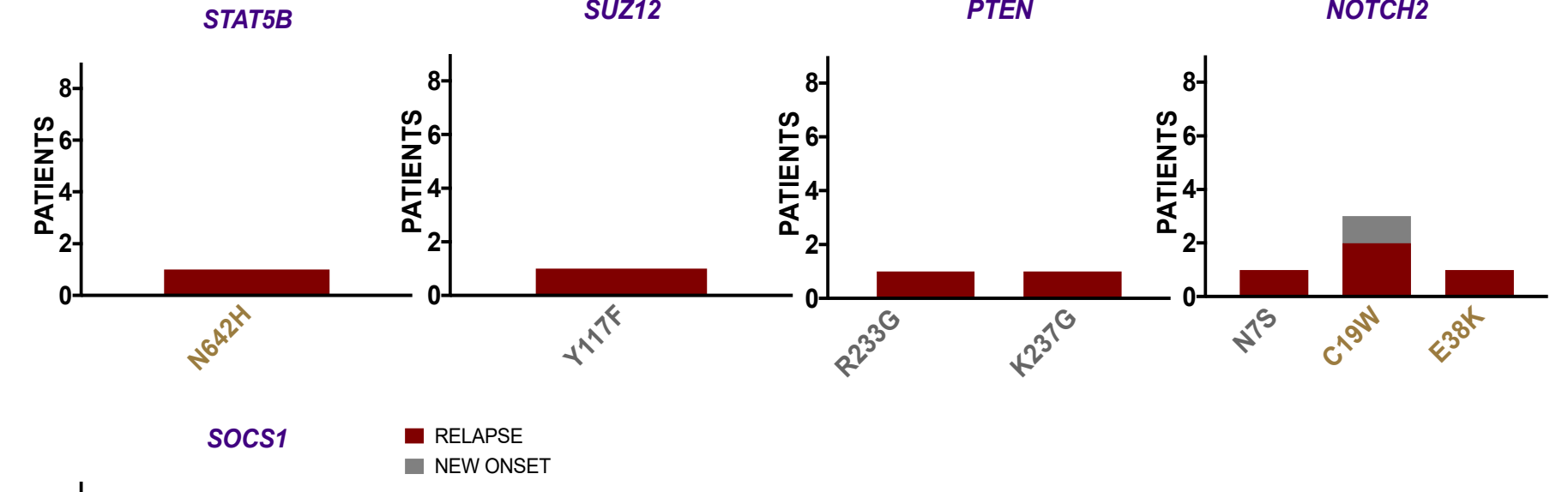

(c)

PTEN (d)

NOTCH2

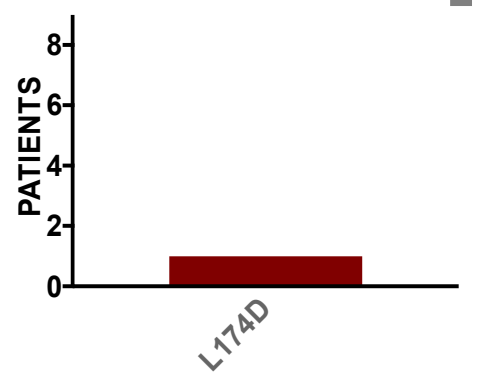

Figure 3. ALL borderland patients harbor tier mutations detected in third-level biomarkers with diagnostic value for leukemia. ALL mutations occur in (a) JAK/STAT, (b) epigenetic, (c) PI3K, and (d) NOTCH2 signaling genes that are used as biomarkers for the diagnosis of leukemias and other hematopoietic malignancies. Tier 3 (gold) and Tier X (grey) mutations are shown occurring in new-onset (grey) and relapse (maroon) ALL patients (superimposed bars) within Level 3 clinically relevant genes (purple).

3.2.4. Prevalence of Borderland Tier Mutations in Hispanic and Non-Hispanic ALL Patients from the TARGET-ALL Phase II Database

The variants at each level of clinically relevant genes were found across patients. Patient 1 harbored the ATM, DNMT3A, TET2, and STAT5B mutations. Others were individual cases including the mutation in SUZ12 in Patient 2, WT1 in Patient 3, SOCS1 in Patient 5, KRAS in Patient 8 and the ROS1 and PTEN mutations in Patient 7. NOTCH2 mutations were dispersed, where Patient 1 contained NOTCH2 N7S and E38K, while Patients 2, 4, 7, 8, and 9 harbored NOTCH2 C19W. Among the select variants reported across clinically relevant genes, those classified as Tier 1-3 are the most plausible in contributing to leukemogenesis in the ALL borderland cohort. Tier $X$ variants require exploratory investigations for oncogenic potential, if not solely for their immediate relevance to clinical application upon any discoveries. Thus, prioritizing the analysis of Tier 1-3 variants in the ALL borderland cohort, ROS1 S1109L, KRAS G12A, WT1 C303 *, STAT5B N642H, NOTCH2 E38K, and NOTCH2 C19W were screened for in the TARGET-ALL Phase II database to investigate their presence across a larger cohort. ROS1 S1109L, WT1 C303 *, and NOTCH2 (C19W, E38K) mutations were not found in the larger cohort, and all occurred as individual cases in the borderland ALL cohort, except for NOTCH2 C19W, which occurred in five of nine patients. KRAS G12A and STAT5B N642H were detected in the TARGET-ALL Phase II database. The frequency of these variants was converted to percent and compared between Hispanic and non-Hispanic white ALL patients (Figure 4). It is worth mentioning that the exact AA change in the borderland cohort was KRAS G12A, while the TARGET-ALL Phase II database included multiple AA changes in KRAS at position 12 (KRAS G12X). The frequency of KRAS G12X in the larger Hispanic cohort was roughly $11 \%$ in contrast to $3 \%$ in non-Hispanic white patients. KRAS G12A occurred in one of nine cases from the ALL borderland cohort, reflecting observations in the database. In contrast, the frequency 
of STAT5B N642H was similar, at roughly $8 \%$ in both Hispanic and non-Hispanic ALL patients. The STAT5B N642H mutation occurred in one of nine cases in the ALL borderland cohort. The detection of this somewhat rare mutation within this small borderland cohort may suggest that further sampling would reveal a more frequent event of this aberration in Hispanic ALL patients, and perhaps those of Mexican-American origin.

\section{PREVALENCE OF MUTATIONS}

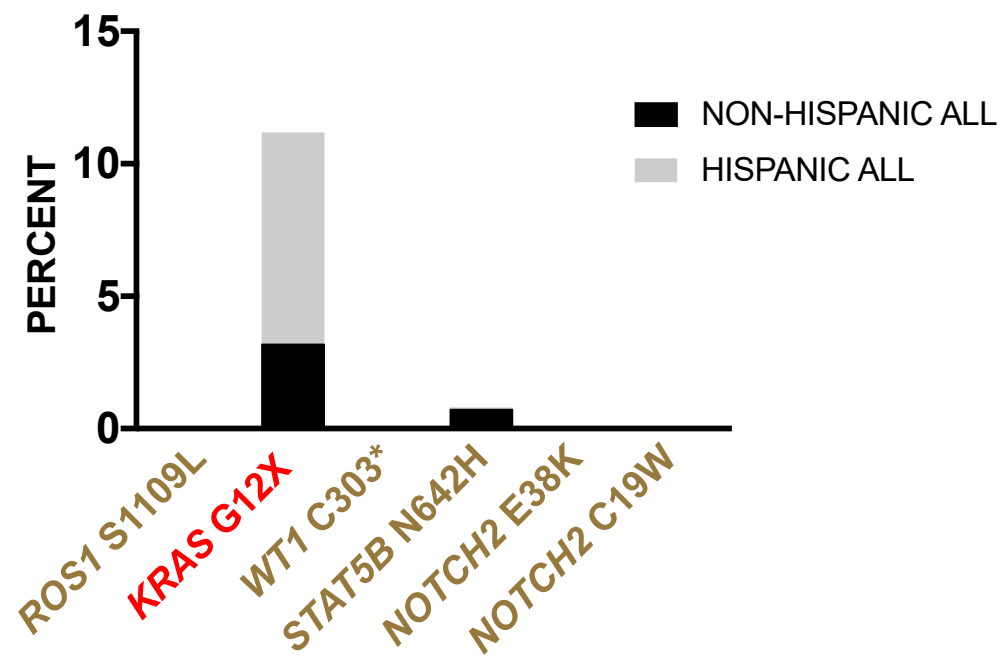

Figure 4. Prevalence of borderland tier mutations in Hispanic and non-Hispanic ALL patients from the TARGET-ALL Phase II database. Variants classified as Tier 1 (red) and Tier 3 (gold) are shown with their frequency in percent (superimposed bars) across Hispanic ALL patients (light gray) and non-Hispanic patients (black) from the TARGET-ALL Phase II database.

\section{Discussion}

The genetic diversity of ALL represents a significant challenge for precision medicine. Here, we explored the mutational landscape of ALL patients from a demographic burdened with leukemia health disparities residing in the borderland. Notable variants were identified within common cancer biomarkers. Specifically, ALL cases contained mutations in potentially actionable genes, including KRAS G12A and ROS1 S1109. KRAS aberrations are an inclusion criterion for ongoing clinical trials in ALL (https: / /www.mycancergenome. org / content/gene/ kras/) (accessed on 07 July 2021). ROS1 S1109L is observed in 0.016\% of the Latino/Admixed American group of the GnomAD database and is classified as a Tier 3 mutation in the COSMIC database. This mutation should be explored for its influence as a predisposing factor in cancer. Another notable variant was WT1 C303 * This gene is recognized as a biomarker with diagnostic value for AML; whether this variant can provide diagnostic value in ALL should be explored. NOTCH2 mutations were frequently observed in the ALL borderland cohort as seen in T-ALL [40]. The NOTCH2 E38K variant was also reported in B-ALL [42]. The NOTCH2 C19W mutation has been reported in angioimmunoblastic $\mathrm{T}$ cell lymphoma [43]. This mutation was detected in both new-onset and relapse cases, and it seems plausible that it may be retained during the progression of ALL and contribute to relapse. An obvious shortcoming is the absence of diagnosis-relapse pair controls; thus, there is no direct verification of the identified variants as somatic. NOTCH2 mutations have diagnostic and prognostic value for certain lymphomas that are predictive of adverse outcomes [44]. Thus, the outcomes of ALL patients harboring NOTCH2 C19W or E38K should be explored. Lastly, the STAT5B N642H variant that confers poor prognosis in T-ALL [37] was also detected in our ALL cohort. Each of these variants was screened against ALL cases within the TARGET-ALL Phase II dataset, which includes a representation of $17 \%$ Hispanics. The KRAS G12X and STAT5B N642H were the only Tier 1-3 variants detected in this database. Interestingly, the occurrence of 
KRAS G12X was similar across Hispanics in both the ALL borderland cohort and the larger database, and three-fold greater than that observed in non-Hispanic whites. The occurrence of STAT5B N642H is likely greater in Hispanic ALL patients from the borderland compared to those within the large database. This inconsistency across datasets may be in part due to the mixed lineage termed Hispanic in the TARGET-ALL Phase II dataset, whereas the Paso del Norte borderland dataset includes Hispanics from primarily Mexican-American origin. Thus, aberrations in the MAPK pathway may be contributing to Hispanic health disparities in ALL, while aberrations in the JAK/STAT pathway may be more relevant to health disparities unique to Mexican-Americans or the borderland. An adequate number of clinical samples from diverse lineages are needed to confirm this interpretation.

In our cohort, we detected the activating STAT5B N642H mutation and SOCS1 L174D that signal in the JAK/STAT pathway. It is important to note that these genes within the JAK/STAT pathway are considered biomarkers within the lowest category of clinical evidence. It is proposed that frequent sampling of diverse ALL patients may change the status of JAK/STAT genes into a higher level of clinical evidence that would be complimented by the targetability of this pathway. In B-ALL subtypes that lack known fusion transcripts, aberrant mutations within the MAPK/RTK and JAK/STAT pathways are targeted [45]. Our cohort may have been diagnosed with similar ALL subtypes, as increased frequency of Ph-like ALL is observed in Hispanics and associated with inferior outcomes [46]. Many of these subtypes are complemented by NGS data to help stratify patients and treatment regimens. Thus, the Hispanic Mexican-origin demographic that experience health disparities in ALL may benefit from NGS efforts in stratification to detect relevant biomarkers that may be overlooked in immunophenotyping and cytogenetics alone. Another pathway harboring mutated genes in our borderland ALL cohort is the chromatin remodeling pathway, where DNMT3A, TET2, WT1, and SUZ12 all influence epigenetic regulation. Epigenetic modulators are frequently mutated in ALL relapse [47,48]. Given the potential to therapeutically target this pathway, tier variants observed in these genes should be further explored for their oncogenic potential in ALL. Collectively, this implies that more work is needed across a diverse population and within high-risk groups to determine new biomarkers and strategies for actionable mutations in ALL.

\section{Conclusions}

Collectively, curated data, largely derived from NGS studies, have aided in providing diagnostic, prognostic, and therapeutic biomarkers for ALL. One major finding included the detection of KRAS G12A and STAT5B N642H in Hispanic ALL borderland patients. Interestingly, the occurrence of KRAS G12A is similar across Hispanics in both the ALL borderland cohort and the TARGET-ALL Phase II dataset, yet greater than that observed in non-Hispanic whites. The occurrence of STAT5B N642H might be greater in Hispanic ALL patients from the borderland compared to those within the database. Thus, aberrations in the MAPK pathway may be contributing to Hispanic health disparities in ALL, while aberrations in the JAK/STAT pathway may be more relevant to health disparities unique to Hispanic Mexican-Americans. KRAS G12A and STAT5B N642H mutations in ALL are associated with relapse and may therefore be contributing to Hispanic health disparities in ALL. Furthermore, the JAK/STAT and chromatin remodeling pathways were found harboring mutations in our ALL borderland cohort. Given that these pathways are theoretically targetable, Tier $\mathrm{X}$ variants within these genes warrant investigation for driving leukemogenesis.

Supplementary Materials: The following are available online at https:/ / www.mdpi.com/article/10 .3390/ijerph18147345/s1, Figure S1: Clinically relevant genes.

Author Contributions: Conceptualization, A.H.G. and R.A.K.; data curation, A.H.G., Y.M.A.-M., J.E.M., E.R.-E. and G.R.; formal analysis, A.H.G., Y.M.A.-M., J.E.M. and J.D.; funding acquisition, R.A.K.; supervision, R.A.K.; writing-original draft, A.H.G., Y.M.A.-M., J.E.M., E.R.-E., G.R. and J.D.; 
writing-review and editing, A.H.G., Y.M.A.-M. and R.A.K. All authors have read and agreed to the published version of the manuscript.

Funding: This research was funded by the National Institute on Minority Health and Health Disparities, a component of the National Institutes of Health (5U54MD007592).

Institutional Review Board Statement: The study was conducted according to the guidelines of the Declaration of Helsinki and approved by the Institutional Review Board of The University of Texas at El Paso (Ethic code 397292-12 and approved 2012).

Informed Consent Statement: Informed consent was obtained from all subjects involved in the study.

Data Availability Statement: Data generated in this study may be accessed from the UTEP Bioinformatics Repository (https: / / datarepo.bioinformatics.utep.edu/getdata?acc=B4U5YED0W0RIE3C) (accessed on 7 July 2021).

Acknowledgments: Special acknowledgment to the staff of the BBRC facilities, including the Bioinformatics Computing Core Laboratory. We would also like to acknowledge the Therapeutically Applicable Research to Generate Effective Treatments (TARGET) initiative, phs000218, managed by the NCI. Information about TARGET can be found at http:/ / ocg.cancer.gov/programs/target (accessed on 1 April 2021).

Conflicts of Interest: The authors declare no conflict of interest.

\section{References}

1. Nguyen, K.; Devidas, M.; Cheng, S.C.; La, M.; Raetz, E.A.; Carroll, W.L.; Winick, N.J.; Hunger, S.P.; Gaynon, P.S.; Loh, M.L.; et al. Factors influencing survival after relapse from acute lymphoblastic leukemia: A Children's Oncology Group study. Leukemia 2008, 22, 2142-2150. [CrossRef]

2. Miller, K.D.; Goding Sauer, A.; Ortiz, A.P.; Fedewa, S.A.; Pinheiro, P.S.; Tortolero-Luna, G.; Martinez-Tyson, D.; Jemal, A.; Siegel, R.L. Cancer Statistics for Hispanics/Latinos, 2018. CA Cancer J. Clin. 2018, 68, 425-445. [CrossRef]

3. Karol, S.E.; Pui, C.H. Personalized therapy in pediatric high-risk B-cell acute lymphoblastic leukemia. Ther. Adv. Hematol. 2020, 11, 2040620720927575. [CrossRef] [PubMed]

4. Arber, D.A.; Orazi, A.; Hasserjian, R.; Thiele, J.; Borowitz, M.J.; Le Beau, M.M.; Bloomfield, C.D.; Cazzola, M.; Vardiman, J.W. The 2016 revision to the World Health Organization classification of myeloid neoplasms and acute leukemia. Blood 2016, 127, 2391-2405. [CrossRef]

5. $\quad$ Roberts, K.G.; Yang, Y.L.; Payne-Turner, D.; Lin, W.; Files, J.K.; Dickerson, K.; Gu, Z.; Taunton, J.; Janke, L.J.; Chen, T.; et al. Oncogenic role and therapeutic targeting of ABL-class and JAK-STAT activating kinase alterations in Ph-like ALL. Blood Adv. 2017, 1, 1657-1671. [CrossRef]

6. Moorman, A.V. New and emerging prognostic and predictive genetic biomarkers in B-cell precursor acute lymphoblastic leukemia. Haematologica 2016, 101, 407-416. [CrossRef] [PubMed]

7. O’Donnell, M.R.; Tallman, M.S.; Abboud, C.N.; Altman, J.K.; Appelbaum, F.R.; Arber, D.A.; Bhatt, V.; Bixby, D.; Blum, W.; Coutre, S.E.; et al. Acute Myeloid Leukemia, Version 3.2017, NCCN Clinical Practice Guidelines in Oncology. J. Natl. Compr. Cancer Netw. 2017, 15, 926-957. [CrossRef] [PubMed]

8. Coccaro, N.; Anelli, L.; Zagaria, A.; Specchia, G.; Albano, F. Next-Generation Sequencing in Acute Lymphoblastic Leukemia. Int. J. Mol. Sci. 2019, 20, 2929. [CrossRef]

9. Loree, J.M.; Anand, S.; Dasari, A.; Unger, J.M.; Gothwal, A.; Ellis, L.M.; Varadhachary, G.; Kopetz, S.; Overman, M.J.; Raghav, K. Disparity of Race Reporting and Representation in Clinical Trials Leading to Cancer Drug Approvals From 2008 to 2018. JAMA Oncol. 2019, e191870. [CrossRef]

10. Dickmann, L.J.; Schutzman, J.L. Racial and Ethnic Composition of Cancer Clinical Drug Trials: How Diverse Are We? Oncologist 2018, 23, 243-246. [CrossRef]

11. Heredia, N.I.; Krasny, S.; Strong, L.L.; Von Hatten, L.; Nguyen, L.; Reininger, B.M.; McNeill, L.H.; Fernandez, M.E. Community Perceptions of Biobanking Participation: A Qualitative Study among Mexican-Americans in Three Texas Cities. Public Health Genom. 2017, 20, 46-57. [CrossRef] [PubMed]

12. Bencomo-Alvarez, A.E.; Gonzalez, M.A.; Rubio, A.J.; Olivas, I.M.; Lara, J.J.; Padilla, O.; Orazi, A.; Corral, J.; Philipovskiy, A.; Gaur, S.; et al. Ethnic and border differences on blood cancer presentation and outcomes: A Texas population-based study. Cancer 2020, 127, 1068-1079. [CrossRef] [PubMed]

13. Chakravarty, D.; Gao, J.; Phillips, S.M.; Kundra, R.; Zhang, H.; Wang, J.; Rudolph, J.E.; Yaeger, R.; Soumerai, T.; Nissan, M.H.; et al. OncoKB: A Precision Oncology Knowledge Base. JCO Precis Oncol. 2017, 2017. [CrossRef] [PubMed]

14. Tate, J.G.; Bamford, S.; Jubb, H.C.; Sondka, Z.; Beare, D.M.; Bindal, N.; Boutselakis, H.; Cole, C.G.; Creatore, C.; Dawson, E.; et al. COSMIC: The Catalogue Of Somatic Mutations In Cancer. Nucleic Acids Res. 2019, 47, D941-D947. [CrossRef] 
15. Jain, N.; Mittendorf, K.F.; Holt, M.; Lenoue-Newton, M.; Maurer, I.; Miller, C.; Stachowiak, M.; Botyrius, M.; Cole, J.; Micheel, C.; et al. The My Cancer Genome clinical trial data model and trial curation workflow. J. Am. Med. Inform. Assoc. 2020, 27, 1057-1066. [CrossRef]

16. Bolger, A.M.; Lohse, M.; Usadel, B. Trimmomatic: A flexible trimmer for Illumina sequence data. Bioinformatics 2014, 30, 2114-2120. [CrossRef]

17. Lavretsky, P.; McInerney, N.R.; Mohl, J.E.; Brown, J.I.; James, H.F.; McCracken, K.G.; Fleischer, R.C. Assessing changes in genomic divergence following a century of human-mediated secondary contact among wild and captive-bred ducks. Mol. Ecol. 2020, 29, 578-595. [CrossRef]

18. Li, H.; Durbin, R. Fast and accurate short read alignment with Burrows-Wheeler transform. Bioinformatics 2009, 25, 1754-1760. [CrossRef]

19. Li, H.; Handsaker, B.; Wysoker, A.; Fennell, T.; Ruan, J.; Homer, N.; Marth, G.; Abecasis, G.; Durbin, R.; Genome Project Data Processing, S. The Sequence Alignment/Map format and SAMtools. Bioinformatics 2009, 25, 2078-2079. [CrossRef]

20. Leung, M.-Y.; Knapka, J.A.; Wagler, A.E.; Rodriguez, G.; Kirken, R.A. OncoMiner: A Pipeline for Bioinformatics Analysis of Exonic Sequence Variants in Cancer. In Big Data Analytics in Genomics; Springer: Cham, Switzerland, 2016.

21. Choi, Y.; Chan, A.P. PROVEAN web server: A tool to predict the functional effect of amino acid substitutions and indels. Bioinformatics 2015, 31, 2745-2747. [CrossRef]

22. Cingolani, P.; Platts, A.; Wang, L.L.; Coon, M.; Nguyen, T.; Wang, L.; Land, S.J.; Lu, X.; Ruden, D.M. A program for annotating and predicting the effects of single nucleotide polymorphisms, SnpEff: SNPs in the genome of Drosophila melanogaster strain w1118; iso-2; iso-3. Fly 2012, 6, 80-92. [CrossRef]

23. Genomes Project, C.; Auton, A.; Brooks, L.D.; Durbin, R.M.; Garrison, E.P.; Kang, H.M.; Korbel, J.O.; Marchini, J.L.; McCarthy, S.; McVean, G.A.; et al. A global reference for human genetic variation. Nature 2015, 526, 68-74. [CrossRef]

24. Purcell, S.; Neale, B.; Todd-Brown, K.; Thomas, L.; Ferreira, M.A.; Bender, D.; Maller, J.; Sklar, P.; de Bakker, P.I.; Daly, M.J.; et al. PLINK: A tool set for whole-genome association and population-based linkage analyses. Am. J. Hum. Genet. 2007, 81, 559-575. [CrossRef]

25. Tabbò, F.; Pizzi, M.; Kyriakides, P.W.; Ruggeri, B.; Inghirami, G. Oncogenic kinase fusions: An evolving arena with innovative clinical opportunities. Oncotarget 2016, 7, 25064-25086. [CrossRef]

26. Uguen, A.; De Braekeleer, M. ROS1 fusions in cancer: A review. Future Oncol 2016, 12, 1911-1928. [CrossRef] [PubMed]

27. Gumy-Pause, F.; Wacker, P.; Sappino, A.P. ATM gene and lymphoid malignancies. Leukemia 2004, 18, 238-242. [CrossRef] [PubMed]

28. Landrum, M.J.; Lee, J.M.; Benson, M.; Brown, G.R.; Chao, C.; Chitipiralla, S.; Gu, B.; Hart, J.; Hoffman, D.; Jang, W.; et al. ClinVar: Improving access to variant interpretations and supporting evidence. Nucleic Acids Res. 2018, 46, D1062-D1067. [CrossRef] [PubMed]

29. Irving, J.; Matheson, E.; Minto, L.; Blair, H.; Case, M.; Halsey, C.; Swidenbank, I.; Ponthan, F.; Kirschner-Schwabe, R.; GroeneveldKrentz, S.; et al. Ras pathway mutations are prevalent in relapsed childhood acute lymphoblastic leukemia and confer sensitivity to MEK inhibition. Blood 2014, 124, 3420-3430. [CrossRef]

30. Oshima, K.; Khiabanian, H.; da Silva-Almeida, A.C.; Tzoneva, G.; Abate, F.; Ambesi-Impiombato, A.; Sanchez-Martin, M.; Carpenter, Z.; Penson, A.; Perez-Garcia, A.; et al. Mutational landscape, clonal evolution patterns, and role of RAS mutations in relapsed acute lymphoblastic leukemia. Proc. Natl. Acad. Sci. USA 2016, 113, 11306-11311. [CrossRef] [PubMed]

31. Bond, J.; Touzart, A.; Leprêtre, S.; Graux, C.; Bargetzi, M.; Lhermitte, L.; Hypolite, G.; Leguay, T.; Hicheri, Y.; Guillerm, G.; et al. DNMT3A mutation is associated with increased age and adverse outcome in adult T-cell acute lymphoblastic leukemia. Haematologica 2019, 104, 1617-1625. [CrossRef]

32. Wang, R.Q.; Chen, C.J.; Jing, Y.; Qin, J.Y.; Li, Y.; Chen, G.F.; Zhou, W.; Li, Y.H.; Wang, J.; Li, D.W.; et al. Characteristics and prognostic significance of genetic mutations in acute myeloid leukemia based on a targeted next-generation sequencing technique. Cancer Med. 2020, 9, 8457-8467. [CrossRef]

33. Krauth, M.T.; Alpermann, T.; Bacher, U.; Eder, C.; Dicker, F.; Ulke, M.; Kuznia, S.; Nadarajah, N.; Kern, W.; Haferlach, C.; et al. WT1 mutations are secondary events in AML, show varying frequencies and impact on prognosis between genetic subgroups. Leukemia 2015, 29, 660-667. [CrossRef] [PubMed]

34. Zhang, P.; Weng, W.W.; Chen, P.; Zhang, Y.; Ruan, J.F.; Ba, D.D.; Xu, W.Q.; Tang, Y.M. Low expression of TET2 gene in pediatric acute lymphoblastic leukemia is associated with poor clinical outcome. Int. J. Lab. Hematol. 2019, 41, 702-709. [CrossRef] [PubMed]

35. Qin, Y.Z.; Jiang, Q.; Xu, L.P.; Jiang, H.; Wang, Y.; Zhao, X.S.; Li, Z.R.; Lai, Y.Y.; Liu, Y.R.; Zhang, X.H.; et al. The prognostic significance of Wilms' tumor gene 1 (WT1) expression at diagnosis in adults with Ph-negative B cell precursor acute lymphoblastic leukemia. Ann. Hematol. 2019, 98, 2551-2559. [CrossRef]

36. Rampal, R.; Figueroa, M.E. Wilms tumor 1 mutations in the pathogenesis of acute myeloid leukemia. Haematologica 2016, 101, 672-679. [CrossRef]

37. Bandapalli, O.R.; Schuessele, S.; Kunz, J.B.; Rausch, T.; Stutz, A.M.; Tal, N.; Geron, I.; Gershman, N.; Izraeli, S.; Eilers, J.; et al. The activating STAT5B N642H mutation is a common abnormality in pediatric T-cell acute lymphoblastic leukemia and confers a higher risk of relapse. Haematologica 2014, 99, e188-e192. [CrossRef] 
38. Ntziachristos, P.; Tsirigos, A.; Vlierberghe, P.V.; Nedjic, J.; Trimarchi, T.; Flaherty, M.S.; Ferres-Marco, D.; da Ros, V.; Tang, Z.; Siegle, J.; et al. Genetic inactivation of the polycomb repressive complex 2 in T cell acute lymphoblastic leukemia. Nat. Med. 2012, 18, 298-302. [CrossRef] [PubMed]

39. Mendes, R.D.; Canté-Barrett, K.; Pieters, R.; Meijerink, J.P. The relevance of PTEN-AKT in relation to NOTCH1-directed treatment strategies in T-cell acute lymphoblastic leukemia. Haematologica 2016, 101, 1010-1017. [CrossRef] [PubMed]

40. Neumann, M.; Vosberg, S.; Schlee, C.; Heesch, S.; Schwartz, S.; Gökbuget, N.; Hoelzer, D.; Graf, A.; Krebs, S.; Bartram, I.; et al. Mutational spectrum of adult T-ALL. Oncotarget 2015, 6, 2754-2766. [CrossRef] [PubMed]

41. Hou, H.A.; Lu, J.W.; Lin, T.Y.; Tsai, C.H.; Chou, W.C.; Lin, C.C.; Kuo, Y.Y.; Liu, C.Y.; Tseng, M.H.; Chiang, Y.C.; et al. Clinicobiological significance of suppressor of cytokine signaling 1 expression in acute myeloid leukemia. Blood Cancer J. 2017, 7, e588. [CrossRef] [PubMed]

42. Takam Kamga, P.; Dal Collo, G.; Midolo, M.; Adamo, A.; Delfino, P.; Mercuri, A.; Cesaro, S.; Mimiola, E.; Bonifacio, M.; Andreini, A.; et al. Inhibition of Notch Signaling Enhances Chemosensitivity in B-cell Precursor Acute Lymphoblastic Leukemia. Cancer Res. 2019, 79, 639-649. [CrossRef] [PubMed]

43. Holst, J.M.; Pedersen, M.B.; Hansen, M.C.; Plesner, T.L.; Frederiksen, H.; Møller, M.; Hamilton-Dutoit, S.; Noergaard, P.; Mortensen, B.K.; Fiore, D.; et al. Shared Genomic Alterations in Patients with Co-Existing Myeloproliferative Neoplasms and Angioimmunoblastic T-Cell Lymphoma. Blood 2019, 134, 2776. [CrossRef]

44. Kiel, M.J.; Velusamy, T.; Betz, B.L.; Zhao, L.; Weigelin, H.G.; Chiang, M.Y.; Huebner-Chan, D.R.; Bailey, N.G.; Yang, D.T.; Bhagat, G.; et al. Whole-genome sequencing identifies recurrent somatic NOTCH2 mutations in splenic marginal zone lymphoma. J. Exp. Med. 2012, 209, 1553-1565. [CrossRef]

45. Messina, M.; Chiaretti, S.; Wang, J.; Fedullo, A.L.; Peragine, N.; Gianfelici, V.; Piciocchi, A.; Brugnoletti, F.; Di Giacomo, F.; Pauselli, S.; et al. Prognostic and therapeutic role of targetable lesions in B-lineage acute lymphoblastic leukemia without recurrent fusion genes. Oncotarget 2016, 7, 13886-13901. [CrossRef]

46. Jain, N.; Roberts, K.G.; Jabbour, E.; Patel, K.; Eterovic, A.K.; Chen, K.; Zweidler-McKay, P.; Lu, X.; Fawcett, G.; Wang, S.A.; et al. Ph-like acute lymphoblastic leukemia: A high-risk subtype in adults. Blood 2017, 129, 572-581. [CrossRef]

47. Kunz, J.B.; Rausch, T.; Bandapalli, O.R.; Eilers, J.; Pechanska, P.; Schuessele, S.; Assenov, Y.; Stutz, A.M.; Kirschner-Schwabe, R.; Hof, J.; et al. Pediatric T-cell lymphoblastic leukemia evolves into relapse by clonal selection, acquisition of mutations and promoter hypomethylation. Haematologica 2015, 100, 1442-1450. [CrossRef] [PubMed]

48. Li, B.; Brady, S.W.; Ma, X.; Shen, S.; Zhang, Y.; Li, Y.; Szlachta, K.; Dong, L.; Liu, Y.; Yang, F.; et al. Therapy-induced mutations drive the genomic landscape of relapsed acute lymphoblastic leukemia. Blood 2020, 135, 41-55. [CrossRef] [PubMed] 